\title{
Relation between Leukocyte Telomere Length and Incident Coronary Heart Disease Events (From the 1995 Canadian Nova Scotia Health Survey)
}

\author{
Siqin Ye, MDa, Jonathan A. Shaffer, $\mathrm{PhD}^{\mathrm{a}}$, Min Suk Kang, $\mathbf{P h D}^{\mathrm{b}}$, Manjunath Harlapur, $\mathbf{M D}^{\mathrm{a}}$, \\ Paul Muntner, PhD $^{c}$, Elissa Epel, MD ${ }^{d}$, Duane Guernsey, PhD $^{\mathrm{e}}$, Joseph E. Schwartz, PhDa,f, \\ Karina W. Davidson, PhDa , Susan Kirkland, PhDg, Lawrence S. Honig, MD, PhD ${ }^{b, h, i}$, and \\ Daichi Shimbo, MDa \\ ${ }^{a}$ Center for Behavioral Cardiovascular Health, Department of Medicine, Columbia University, New \\ York, New York, USA \\ 'Taub Institute for Research on Alzheimer's Disease and the Aging Brain, Columbia University, \\ New York, New York, USA \\ 'Department of Epidemiology, University of Alabama at Birmingham, Birmingham, Alabama, USA \\ dDepartment of Psychiatry, University of California, San Francisco, California, USA \\ eDepartment of Pathology, Dalhousie University, Halifax, Nova Scotia, Canada \\ fDepartment of Psychiatry, Stony Brook University, Stony Brook, New York \\ gDepartment of Community Health \& Epidemiology, Dalhousie University, Halifax, Nova Scotia, \\ Canada \\ h'Gertrude H. Sergievsky Center, Columbia University, New York, NY, USA \\ 'Department of Neurology, Columbia University Medical Center, New York, NY, USA
}

\section{Abstract}

\begin{abstract}
Leukocyte telomere length has been proposed as a biomarker of cellular aging and atherosclerosis. We sought to determine whether leukocyte telomere length is independently associated with incident coronary heart disease (CHD) in the general population. Telomere length was measured using a polymerase chain reaction method for participants enrolled in the 1995 Nova Scotia Health Survey $(\mathrm{n}=1,917)$. The primary endpoint was first occurrence of fatal and non-fatal CHD events. During a mean follow-up of 8.7 years, 164 fatal or non-fatal CHD events occurred. Compared to participants in the longest tertile of telomere length, those in the middle and shortest tertiles had increased incidence of CHD events (6.2,11.2 and 12.2 per 1000 person-years, respectively). After adjustment for demographics, traditional risk factors and inflammatory markers including hs-CRP, IL-6, and sICAM-1, those in the middle tertile had significantly elevated risk for incident CHD (hazard ratio [HR] 1.63, 95\% CI 1.07-2.51, $\mathrm{p}=0.02$ ) compared to the longest tertile, whereas the risk for those in the shortest tertile was non-significantly elevated (HR 1.25, 95\% CI 0.82-1.90,
\end{abstract}

\footnotetext{
(C) 2013 Excerpta Medica, Inc. All rights reserved.

Correspondence to: Daichi Shimbo, Columbia University Medical Center, 622 West $168^{\text {th }}$ Street, PH 9-310, New York, NY 10032 , Phone: (212) 342-4490, Fax: (646) 304-7003, ds2231@ columbia.edu.

Publisher's Disclaimer: This is a PDF file of an unedited manuscript that has been accepted for publication. As a service to our customers we are providing this early version of the manuscript. The manuscript will undergo copyediting, typesetting, and review of the resulting proof before it is published in its final citable form. Please note that during the production process errors may be discovered which could affect the content, and all legal disclaimers that apply to the journal pertain.
} 
$\mathrm{p}=0.30$ ). In conclusion, these findings do not support a linear association between leukocyte telomere length and incident CHD risk in the general population.

\section{Keywords}

coronary heart disease; telomere; risk prediction

\section{Introduction}

Telomere length has been proposed as a novel biomarker for vascular aging and for CHD onset. ${ }^{1}$ In 2003, Cawthon et al. reported that shorter leukocyte telomere length was associated with an increased age-adjusted risk for cardiovascular mortality in a convenience sample of 143 initially healthy individuals. ${ }^{2}$ Since that report, discordant findings have been published with some studies showing weak or no association between leukocyte telomere length and cardiovascular risk, ${ }^{3-7}$ and others suggesting a moderate to strong association. ${ }^{1,8-12}$ These studies had notable limitations, such as restricting enrollment to the very elderly leading to possible survival bias ${ }^{3,4,6,7}$, inclusion of participants with prior CHD who are already at increased risk for subsequent events, $3,6,8,12$ and limited adjustment for important confounders such as traditional CHD risk factors. ${ }^{3,4,7,8}$ Few studies have been population-based, further limiting the generalizability of their findings. The extent to which leukocyte telomere length constitutes a valid biomarker for incident CHD events in the general population is thus unclear. To address this, we examined whether shorter leukocyte telomere length was associated with the development of incident CHD events in participants enrolled in the 1995 Nova Scotia Health Survey (NSHS95).

\section{Patients and Methods}

The NSHS95 is a population-based survey implemented by Heart Health Nova Scotia and the Nova Scotia Department of Health. Study participants consisted of non-institutionalized, non-pregnant Nova Scotians, age 18 years or older, and listed in the registry of the national health insurance plan. Of 4,500 targeted participants, a total of 3,227 provided informed consent and were enrolled. The overall recruitment percentage (72\%) is comparable to other large health surveys, and weights applied from propensity score analyses revealed no meaningful response bias. ${ }^{13}$ The current study was approved by the institutional review boards of Dalhousie University, Halifax, Nova Scotia, and Columbia University Medical Center, New York, NY.

For this analysis, we excluded 1,310 participants as follows (Figure 1): 244 participants with prior history of CHD, as determined by claims records for the 5 years preceding the baseline survey using International Classification of Diseases, Ninth Revision (ICD-9) codes 410.x through 414.x (myocardial infarction, acute or chronic ischemic heart disease, angina); 295 participants who did not provide permission for ascertainment of cardiac outcomes; 757 participants who did not have blood samples for telomere length assay; and 14 participants who granted permission but did not have available outcome data. The final cohort thus consisted of 1,917 participants. Compared to the 1,917 participants who had a blood sample for telomere length assessment and had available outcome data, there was no significant difference in age $(\mathrm{p}=0.20)$, sex $(\mathrm{p}=0.52)$, and Framingham risk score $(\mathrm{p}=0.92)$ as defined below for participants who did not have telomere length assessment and/or outcome data $(\mathrm{n}=771)$.

From March through November of 1995, a group of trained nurses contacted eligible individuals and interviewed those who agreed to participate. Those who were interviewed 
also visited a health care clinic approximately 1 week after. At the clinic visit, height and weight were measured, and a comprehensive set of cardiovascular risk factors and full medical history were assessed. A fasting blood sample was obtained and processed for plasma and buffy coat samples. Medication use was recorded during a home visit.

DNA was extracted from frozen buffy coat samples. Average telomere length was determined by a real-time polymerase chain reaction (PCR) method modified from that of Cawthon et al. ${ }^{2,14,15}$ Real-time PCR was performed using a CFX384 thermocycler (Biorad, Richmond, CA). The assay method was optimized for use of both telomere (T) and single copy gene (S) amplifications on the same 384-well plate, with reference standard DNA samples on each plate. Test DNA samples each underwent two triplicate PCR reactions, with use of calibrator samples for correction of inter-plate variability. Amplification primers for telomeres included $\mathrm{T}_{\text {for }}$ : ${ }^{\text {'- }}$ CGGTTTGTTTGGGTTTGGGTTTGGGTTTGGGTTTGGGTT-3' and $\mathrm{T}_{\text {rev }}$ : 5'GGCTTGCCTTACCCTTACCCTTACCCTTACCCTTACCCT-3', and for single copy gene (beta-globin) $\mathrm{S}_{\text {for }}$ 5'-GCTTCTGACACAACTGTGTTCACTAGC-3' and $\mathrm{S}_{\text {rev }} 5^{\prime}$ 'CACCAACTTCATCCACGTTCACC- 3 '. Thermocycling parameters were $95^{\circ} \mathrm{C} \times 10 \mathrm{~min}$ activation, followed by 34 cycles of $95^{\circ} \mathrm{C} \times 15 \mathrm{sec}$, and $55^{\circ} \mathrm{C} \times 120 \mathrm{sec}$. The assay coefficient of variance was 5-8\%. Since the T/S ratio depends on particular DNA standards used, T/S ratios were converted to telomere base pairs (bp) using a formula $(\mathrm{bp}=(1,585 * \mathrm{~T} / \mathrm{S}$ ratio $)+$ 3,582 ) derived from co-analysis of 19 selected DNA samples (correlation coefficient $r=$ $0.90)^{14}$ with both PCR and terminal restriction fragment methods (non-radioactive TeloTAGGG Telomere Length, Roche Diagnostics, Mannheim, Germany). Because telomere base pairs are calculated from $\mathrm{T} / \mathrm{S}$ ratio in a linear fashion, tertiles of telomere length and all resulting statistics were identical between the two measurements. In this analysis, we present telomere length results in base pairs, although caution should be used in comparing absolute telomere length measurements between studies, because of differing methodologies.

Participants' age and sex were recorded from the provincial health insurance registry and verified by the interviewer. Body mass index (BMI) was calculated as weight in kilograms divided by height in meters squared. Systolic blood pressure and diastolic blood pressure were measured using manual sphygmomanometers. Total cholesterol, high-density lipoprotein (HDL) cholesterol, and triglyceride levels were assayed from plasma samples, and low-density lipoprotein (LDL) cholesterol was calculated using the Friedewald formula. ${ }^{16}$ History of diabetes was ascertained by self-report. Those who reported smoking currently or in the past year were considered smokers. Framingham risk score was calculated using age, sex, total and HDL cholesterol levels, systolic blood pressure, and history of diabetes and cigarette smoking. ${ }^{17}$ Lipid lowering medication use was defined as the use of statins, fibrates, bile acid sequestrants, or nicotinic acid. Physical activity was assessed using the Paffenbarger Scale. ${ }^{18}$ Three inflammatory markers, high sensitivity Creactive protein (hs-CRP), interleukin-6 (IL-6), and soluble intercellular adhesion molecule-1 (sICAM-1), were measured from plasma samples. hs-CRP was measured using a latex-enhanced immunonephelometry assay (Cardiophase BN II; Dade Behring, New Castle, Delaware). IL-6 was assessed using a high-sensitivity enzyme-linked immunosorbent assay kit (Quantikine HS IL-6; R\&D Systems, Minneapolis, Minnesota), and sICAM-1 using a commercially available enzyme-linked immunosorbent assay kit (R\&D Systems, Minneapolis, Minnesota). Additional details of study procedures and biomarkers assessments have been published previously. ${ }^{19}$

The primary outcome measure was time-to-first occurrence of fatal or nonfatal CHD event, as determined from hospital discharge codes (ICD- 9 codes 410 through 414 and ICD-10 codes I21-I25) and cause of death listed on death certificates. In Canada, the available 
medical care utilization data included nearly all hospital care delivered to the survey participants. The ICD codes were gathered from the provincial hospital discharge database for a 10-year period following the date of the baseline assessment. For non-fatal CHD events, personnel who performed abstraction were trained and certified, and met regularly with a data quality committee from the Department of Health (Nova Scotia) to ensure accuracy and to adjudicate data entry irregularities. In Canada, Statistics Canada keeps vital statistics at the national level, capturing all deaths including those that occurred at home. Causes of death were coded using ICD codes through a nationally consistent process. For secondary analyses, we also examined the combined endpoint of fatal or non-fatal CHD events or all-cause mortality, as well as all-cause mortality only, non-fatal CHD events only, and fatal CHD events only.

For the primary analysis, based on prior studies, the exposure variable was specified a priori as tertiles of telomere length. 1,6,9,12 Baseline demographics and cardiovascular risk factors as well as levels of inflammatory markers were calculated by tertiles of telomere length. Testing for trend of association of baseline characteristics with increasing tertiles of telomere length was performed using Goodman and Kruskal's gamma statistic for categorical variables, and chi-square statistic from linear regression for continuous variables. The association between log-transformed telomere length (due to skewed distribution) and age was assessed using linear regression. To determine the relationship between telomere length and incident CHD events, three Cox proportional hazards regression models were constructed to estimate the hazard ratios (HR) of having telomere length in the middle and shortest tertiles as compared to the longest tertile (reference group). Model 1 adjusted only for age and sex; model 2 adjusted for variables in model 1 plus BMI, Framingham risk score as a continuous variable, use of lipid lowering medications, and physical activity; model 3 adjusted for variables in model 2 , plus inflammatory biomarker levels including logtransformed hs-CRP and log-transformed IL-6 (both due to skewed distribution), and sICAM-1. Next, secondary end points (the composite of fatal and non-fatal CHD events and all-cause mortality, all-cause mortality only, non-fatal CHD events only, and fatal CHD events only) were used as outcome measures in fully-adjusted models that included all covariates in Model 3. Because telomere biology may differ between men and women, ${ }^{20}$ sensitivity analyses were performed using tertiles stratified by sex. For each model, assumptions of proportional hazards were verified with a formal significance test based on Schoenfeld residuals. ${ }^{21} \mathrm{~A}$ test of non-linearity was conducted by considering tertiles of telomere length as both linear and quadratic terms while adjusting for all other covariates in Model 3. All statistical analyses were performed using STATA version 10.0 (StataCorp, College Station, Texas) and SPSS version 18.0 (IBM, Chicago, Illinois).

\section{Results}

For the 1,917 participants included for this analysis, the mean (SD) age was 46.6 (18.4) years, and $51 \%$ were women. Tests for linear trends demonstrated that participants in shorter tertiles of telomere length were older, less likely to be smokers, had higher systolic and diastolic blood pressures, higher Framingham risk score and risk categories, higher levels of LDL, and higher levels of hs-CRP (Table 1). Log telomere length was inversely correlated with age $\left(\mathrm{r}=-0.22\right.$ and $\left.\mathrm{R}^{2}=0.049, \mathrm{p}<0.001\right)$. For each decade increase in age, a $1.3 \%(95 \%$ $\mathrm{CI}, 1.0 \%$ to $1.5 \%$ ) decrease in telomere length was observed ( $\mathrm{p}<0.001)$.

During a mean follow-up period of 8.7 years, 164 fatal or non-fatal CHD events occurred. The incidence of fatal and non-fatal CHD events was higher for participants in the middle and shortest tertiles, compared to those in the longest tertile (Table 2 and Figure 2). After adjusting for age and sex, participants in the middle tertile continued to have a significantly increased risk of incident CHD (HR 1.63, 95\% CI 1.08-2.47; $\mathrm{p}=0.02$ ), while participants in 
the shortest tertile had a non-significantly increased risk (HR 1.22, 95\% CI 0.81-1.83; $\mathrm{p}=0.35$ ) (Table 2, Model 1). These relationships were similar after adjustment for BMI, Framingham risk score, baseline lipid-lowering medication use including statins, and physical activity (Table 2, Model 2) and further adjustment for inflammatory biomarkers including hs-CRP, IL-6, and sICAM-1 (Table 2, Model 3). Non-linearity of the association of tertiles of telomere length to incident CHD events was demonstrated using a model that included tertiles of telomere length as both linear and quadratic terms $(\mathrm{p}=0.028)$ in addition to all other covariates in Model 3.

Compared to participants in the longest tertile, participants in the middle and shortest tertiles of telomere length had increased number for all secondary endpoints (Table 3). However, after adjustment for demographic factors, traditional risk factors and inflammatory markers, the HRs for secondary endpoints were non-significant, with the exception of non-fatal CHD events, for which the risk was significantly increased for participants in the middle tertile of telomere length compared to those in the longest tertile. The HRs for participants in the middle tertile were non-significantly higher than those for the shortest tertile for all other end points.

Sensitivity analyses were performed using tertiles of telomere length stratified by sex. After full adjustment, the pattern of results differed by sex, although associations for each tertile were not statistically significant. For men, the adjusted HRs for incident CHD events were 1.41 (95\% CI 0.82-2.42; $\mathrm{p}=0.82$ ) for those in the middle tertile of telomere length and 0.94 (95\% CI 0.55-1.61; $\mathrm{p}=0.61$ ) for those in the shortest tertile. However, for women, the adjusted HRs for incident CHD events were similar for both those in the middle (HR 1.72, 95\% CI 0.85-3.48; $\mathrm{p}=0.13$ ) and those in the shortest tertiles (HR 1.72, 95\% CI 0.89-3.36; $\mathrm{p}=0.11$ ), compared to those in the longest tertile.

\section{Discussion}

There are several findings from our analysis of the relationship between telomere length and risk of incident CHD in this population-based cohort. First, in an unadjusted model, shorter telomere length was associated with an increased risk of fatal and non-fatal incident CHD events. However, after adjustment for demographics, traditional cardiovascular risk factors and inflammatory markers, there was a non-linear association of tertiles of telomere length with incident CHD events, with participants in the middle tertile of telomere length having significantly increased risk of incident CHD compared with those in the longest tertile, while those in the shortest tertile did not. Lastly, the pattern of increased CHD risk may differ by sex. In contrast to men, elevated CHD risk was observed for both those in the middle and shortest tertiles of telomere length in women, although these findings did not reach statistical significance.

Previous reports on the relationship between telomere length and cardiac outcomes have produced inconsistent results. Many of the studies that failed to demonstrate a relationship between telomere length and cardiovascular risk exclusively enrolled very elderly participants, with mean ages ranging from 75 to 90 years. ${ }^{3,6}$ Given that telomere length decreases with age, restricting enrollment to the very elderly may exclude individuals with shorter telomere lengths who have already died, resulting in a survivor bias. In contrast, previous studies that included participants with a broader age range as in our study generally found that shorter telomere length being associated with increased cardiovascular risk, ${ }^{8,12}$ though results from a very large population-based study $(n=19,838)$ showed that the strength of this association was modest. ${ }^{22}$ 
It is unclear why the middle tertile of telomere length had a stronger association with CHD events than the shortest tertile in our sample. One explanation is that competing risks from non-cardiovascular deaths may have played a role. Previous studies have shown that individuals with the shortest telomere lengths are at increased risk for incident cancers and also infectious deaths, ${ }^{2,23,24}$ suggesting that the type of disease outcomes may vary by the severity of telomere shortening. It is thus possible that participants in the shortest tertile of telomere length in our study were affected by non-CHD outcomes. Furthermore, Epel et al. hypothesized that competing risks such as cancer or infectious causes of death may operate more strongly in men, ${ }^{10}$ suggesting a biological reason for the potential sex differences we observed. Future studies are needed explore differential predictive values of shorter telomere lengths in men and women, as well as the relationship between telomere length and different causes of death.

Along with the current state of knowledge, our results suggest that telomere length may not have the "ideal" characteristics of a disease-specific biomarker for predicting incident CHD events. $^{25,26}$ Telomere length may have a complex, non-linear relationship with incident $\mathrm{CHD}$ events that is affected by competing risks from other disease processes. In addition, the relatively small decrement in telomere length with increasing age implies that small measurement variation in assays may have a disproportionate effect in models that use telomere length for risk prediction. ${ }^{27}$ Thus, from these results, telomere length cannot be used as a biomarker that has specific "cut-points" for determination of CHD risk. Additional studies would be required to demonstrate the incremental value of telomere length assessment over traditional risk factors.

Our study has several strengths. We used a relatively large, provincially-representative, population-based sample to investigate the relationship between telomere length and the risk of incident CHD. In addition to traditional risk factors, we also assessed inflammatory biomarkers as covariates. We were also able to achieve complete capture of CHD outcomes through centralized databases. There are also several limitations. Although we measured leukocyte telomere length, it is possible that telomere length of specific leukocyte subpopulations or of cardiac or vascular cells might correlate better with cardiac risk. In addition, a single measurement of telomere length cannot indicate whether longitudinal changes in telomere length may better predict CHD risk. ${ }^{10,28}$ Finally, the number of CHD events in this sample was relatively small, and we did not have data on non-CHD disease processes. As such, we could not evaluate the effect of competing risks from non-CHD outcomes.

\section{Acknowledgments}

Supported by NIH grants HL-091099 and HL-084034 from the National Heart, Lung, and Blood Institute, Bethesda, Maryland, and by the National Health and Welfare of Canada, Ottawa, Ontario; the Nova Scotia Department of Health, Halifax, Nova Scotia; and the Heart and Stroke Foundation of New Brunswick, St. John, New Brunswick. Dr. Ye is supported by an American College of Cardiology / Merck Foundation Fellowship Award and by NIH grant T32HL007854-16. Dr. Honig also receives support from the Alzheimer Association, the Alzheimer's Disease Drug Discovery Foundation, NIH/NIA grant P50AG008702, the Henry Panasci Fund, and the Taub Institute. Dr. Epel is a co-founder of Telomere Health, Inc., which offers commercial telomere length measurement.

\section{References}

1. Brouilette SW, Moore JS, McMahon AD, Thompson JR, Ford I, Shepherd J, Packard CJ, Samani NJ. Telomere length, risk of coronary heart disease, and statin treatment in the West of Scotland Primary Prevention Study: a nested case-control study. Lancet. 2007; 369:107-114. [PubMed: 17223473] 
2. Cawthon RM, Smith KR, O'Brien E, Sivatchenko A, Kerber RA. Association between telomere length in blood and mortality in people aged 60 years or older. Lancet. 2003; 361:393-395. [PubMed: 12573379]

3. Martin-Ruiz CM, Gussekloo J, van Heemst D, von Zglinicki T, Westendorp RG. Telomere length in white blood cells is not associated with morbidity or mortality in the oldest old: a population-based study. Aging Cell. 2005; 4:287-290. [PubMed: 16300480]

4. Bischoff C, Petersen HC, Graakjaer J, Andersen-Ranberg K, Vaupel JW, Bohr VA, Kolvraa S, Christensen K. No association between telomere length and survival among the elderly and oldest old. Epidemiology. 2006; 17:190-194. [PubMed: 16477260]

5. Njajou OT, Hsueh WC, Blackburn EH, Newman AB, Wu SH, Li R, Simonsick EM, Harris TM, Cummings SR, Cawthon RM. Association between telomere length, specific causes of death, and years of healthy life in health, aging, and body composition, a population-based cohort study. J Gerontol A Biol Sci Med Sci. 2009; 64:860-864. [PubMed: 19435951]

6. Houben JM, Giltay EJ, Rius-Ottenheim N, Hageman GJ, Kromhout D. Telomere length and mortality in elderly men: the Zutphen Elderly Study. J Gerontol A Biol Sci Med Sci. 2011; 66:3844. [PubMed: 20889650]

7. Strandberg TE, Saijonmaa O, Tilvis RS, Pitkala KH, Strandberg AY, Miettinen TA, Fyhrquist F. Association of telomere length in older men with mortality and midlife body mass index and smoking. J Gerontol A Biol Sci Med Sci. 2011; 66:815-820. [PubMed: 21697500]

8. Fitzpatrick AL, Kronmal RA, Gardner JP, Psaty BM, Jenny NS, Tracy RP, Walston J, Kimura M, Aviv A. Leukocyte Telomere Length and Cardiovascular Disease in the Cardiovascular Health Study. American Journal of Epidemiology. 2007; 165:14-21. [PubMed: 17043079]

9. Honig LS, Schupf N, Lee JH, Tang MX, Mayeux R. Shorter telomeres are associated with mortality in those with APOE epsilon4 and dementia. Ann Neurol. 2006; 60:181-187. [PubMed: 16807921]

10. Epel ES, Merkin SS, Cawthon R, Blackburn EH, Adler NE, Pletcher MJ, Seeman TE. The rate of leukocyte telomere shortening predicts mortality from cardiovascular disease in elderly men. Aging (Albany NY). 2009; 1:81-88. [PubMed: 20195384]

11. Zee RY, Michaud SE, Germer S, Ridker PM. Association of Shorter Mean Telomere Length with Risk of Incident Myocardial Infarction: A Prospective, Nested Case-Control Approach. Clin Chim Acta. 2009; 403:139-141. [PubMed: 19217888]

12. Willeit P, Willeit J, Brandstatter A, Ehrlenbach S, Mayr A, Gasperi A, Weger S, Oberhollenzer F, Reindl M, Kronenberg F, Kiechl S. Cellular aging reflected by leukocyte telomere length predicts advanced atherosclerosis and cardiovascular disease risk. Arterioscler Thromb Vasc Biol. 2010; 30:1649-1656. [PubMed: 20508208]

13. Lawson, B. Evaluation of non-response bias in the Nova Scotia Health Survey 1995. Halifax, Nova Scotia, Canada: Dalhousie University; 1999.

14. Honig LS, Kang MS, Schupf N, Lee JH, Mayeux R. Association of Shorter Leukocyte Telomere Repeat Length With Dementia and Mortality. Arch Neurol. 2012:1332-1339. [PubMed: 22825311]

15. Shaffer JA, Epel E, Kang MS, Ye S, Schwartz JE, Davidson KW, Kirkland S, Honig LS, Shimbo D. Depressive Symptoms Are Not Associated with Leukocyte Telomere Length: Findings from the Nova Scotia Health Survey (NSHS95), a Population-Based Study. PLoS One. 2012; 7:e48318. [PubMed: 23133583]

16. Friedewald WT, Levy RI, Fredrickson DS. Estimation of the concentration of low-density lipoprotein cholesterol in plasma, without use of the preparative ultracentrifuge. Clin Chem. 1972; 18:499-502. [PubMed: 4337382]

17. Wilson PW, D'Agostino RB, Levy D, Belanger AM, Silbershatz H, Kannel WB. Prediction of coronary heart disease using risk factor categories. Circulation. 1998; 97:1837-1847. [PubMed: 9603539]

18. Paffenbarger RS Jr, Wing AL, Hyde RT. Physical activity as an index of heart attack risk in college alumni. Am J Epidemiol. 1978; 108:161-175. [PubMed: 707484]

19. Davidson KW, Schwartz JE, Kirkland SA, Mostofsky E, Fink D, Guernsey D, Shimbo D. Relation of inflammation to depression and incident coronary heart disease (from the Canadian Nova Scotia 
Health Survey [NSHS95] Prospective Population Study). Am J Cardiol. 2009; 103:755-761. [PubMed: 19268727]

20. Moller P, Mayer S, Mattfeldt T, Muller K, Wiegand P, Bruderlein S. Sex-related differences in length and erosion dynamics of human telomeres favor females. Aging (Albany NY). 2009; 1:733-739. [PubMed: 20195387]

21. Grambsch P, Therneau T. Proportional hazards tests and diagnostics based on weighted residuals. Biometrika. 1994; 81:515-526.

22. Weischer M, Bojesen SE, Cawthon RM, Freiberg JJ, Tybjaerg-Hansen A, Nordestgaard BG. Short telomere length, myocardial infarction, ischemic heart disease, and early death. Arterioscler Thromb Vasc Biol. 2012; 32:822-829. [PubMed: 22199369]

23. Willeit P, Willeit J, Mayr A, Weger S, Oberhollenzer F, Brandstatter A, Kronenberg F, Kiechl S. Telomere length and risk of incident cancer and cancer mortality. JAMA. 2010; 304:69-75. [PubMed: 20606151]

24. Fitzpatrick AL, Kronmal RA, Kimura M, Gardner JP, Psaty BM, Jenny NS, Tracy RP, Hardikar S, Aviv A. Leukocyte telomere length and mortality in the Cardiovascular Health Study. J Gerontol A Biol Sci Med Sci. 2011; 66:421-429. [PubMed: 21289018]

25. Vasan RS. Biomarkers of Cardiovascular Disease. Circulation. 2006; 113:2335-2362. [PubMed: 16702488]

26. Wang TJ. Assessing the role of circulating, genetic, and imaging biomarkers in cardiovascular risk prediction. Circulation. 2011; 123:551-565. [PubMed: 21300963]

27. Aviv A, Valdes AM, Spector TD. Human telomere biology: pitfalls of moving from the laboratory to epidemiology. Int J Epidemiol. 2006; 35:1424-1429. [PubMed: 16997848]

28. Kroenke CH, Pletcher MJ, Lin J, Blackburn E, Adler N, Matthews K, Epel E. Telomerase, telomere length, and coronary artery calcium in black and white men in the CARDIA study. Atherosclerosis. 2012; 220:506-512. [PubMed: 22178426] 


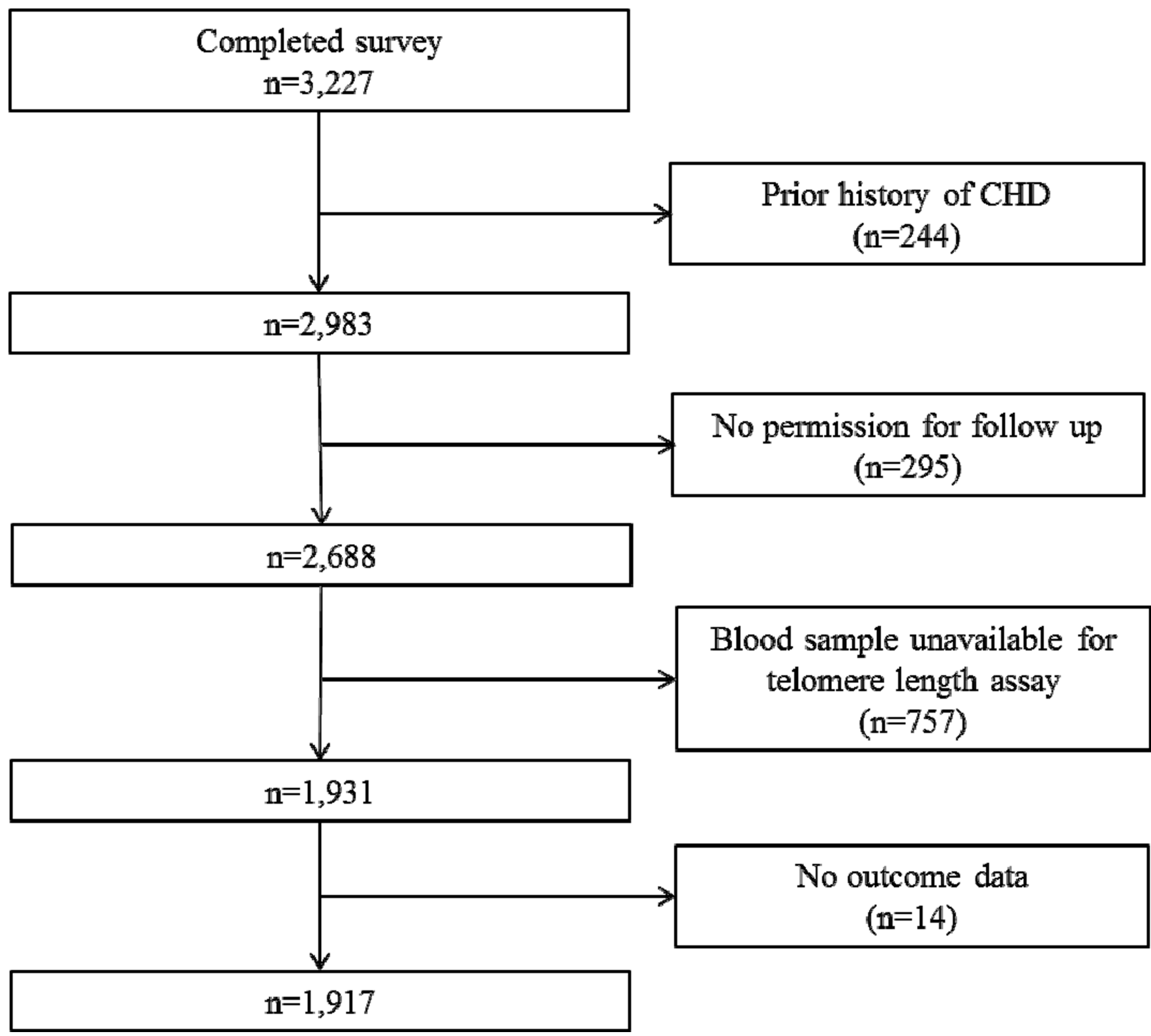

Figure 1. Participants available for analysis of telomere length in the 1995 Nova Scotia Health Survey 


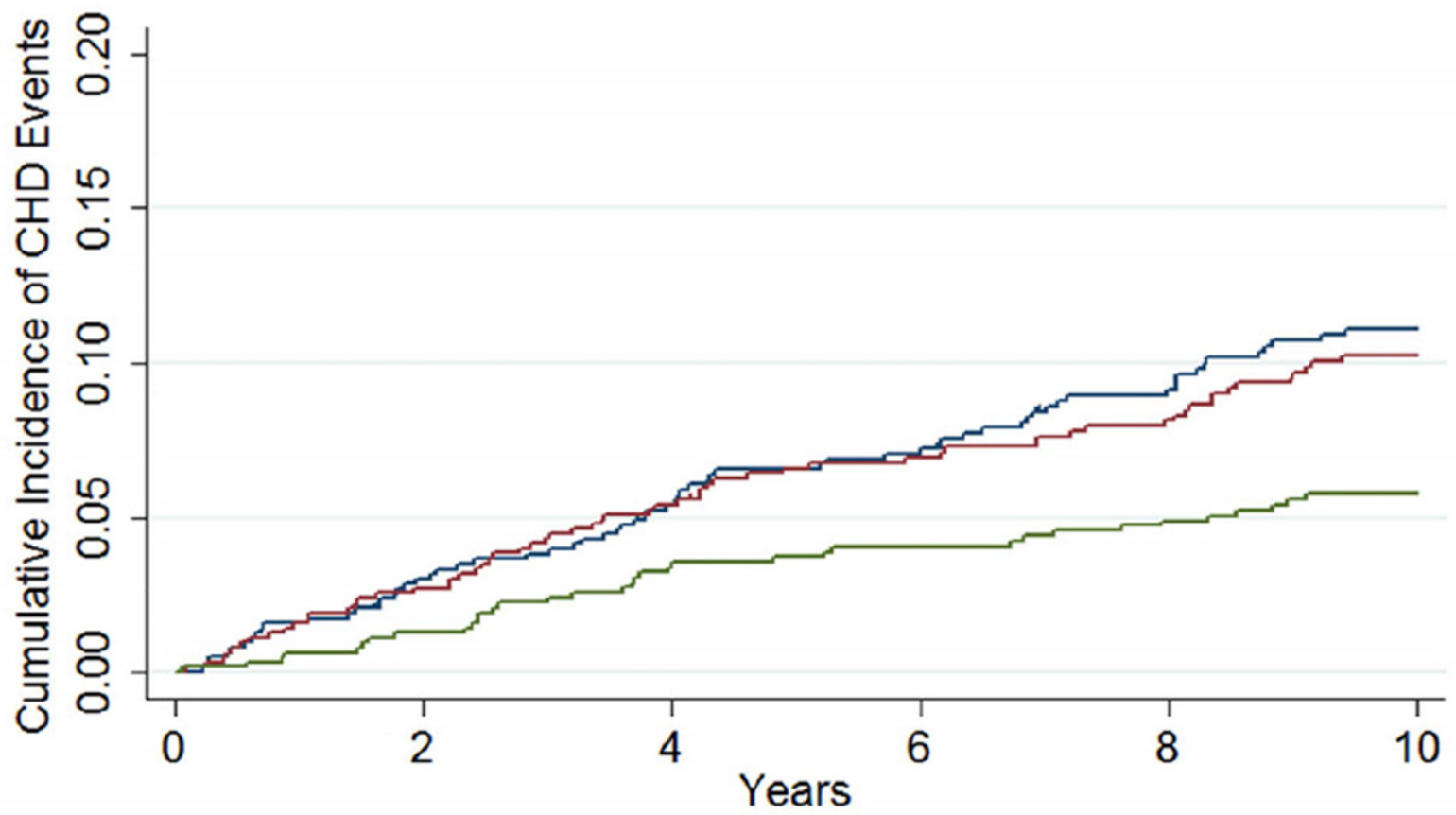

Number at risk

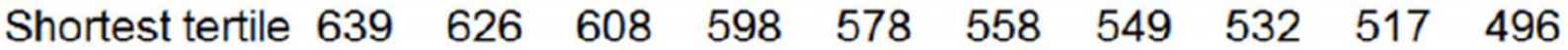

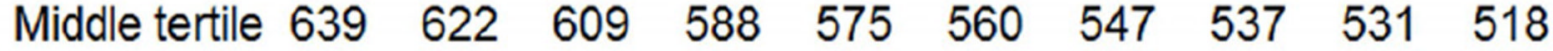
Longest tertile $\begin{array}{llllllllll}639 & 632 & 620 & 608 & 587 & 572 & 560 & 554 & 547 & 533\end{array}$

\section{Shortest tertile \\ Middle tertile Longest tertile}

Figure 2. Cumulative incidence of fatal and non-fatal CHD events, by tertiles of telomere length (in kilo-bases) 
Table 1

Baseline characteristics of 1,917 participants by tertiles of telomere length (in kilo-bases)

\begin{tabular}{|c|c|c|c|c|}
\hline \multirow[b]{2}{*}{ Telomere length (kilo-bases) } & \multicolumn{3}{|c|}{ Tertiles of telomere length } & \multirow[b]{3}{*}{$P$ value } \\
\hline & $\begin{array}{l}5.5 \text { to } 8.2 \\
(\mathrm{n}=639)\end{array}$ & $\begin{array}{c}5.0 \text { to }<5.5 \\
(n=639)\end{array}$ & $\begin{array}{c}4.1 \text { to }<5.0 \\
(\mathrm{n}=639)\end{array}$ & \\
\hline Characteristics & & & & \\
\hline Telomere-to-single copy gene ratio & 1.20 to 2.90 & 0.92 to $<1.20$ & 0.30 to $<0.92$ & \\
\hline Age (years) & $41.7(18.0)$ & $46.1(17.8)$ & $51.8(18.1)$ & $<0.001$ \\
\hline Female & $330(51.6 \%)$ & $340(53.2 \%)$ & $308(48.2 \%)$ & 0.11 \\
\hline Smokers & $193(30.2 \%)$ & $160(25.0 \%)$ & $158(24.7 \%)$ & 0.01 \\
\hline Body mass index $\left(\mathrm{kg} / \mathrm{m}^{2}\right)$ & $26.6(5.4)$ & $27.2(6.1)$ & $27.1(5.0)$ & 0.10 \\
\hline Diabetes mellitus & $21(3.3 \%)$ & $26(4.1 \%)$ & $23(3.6 \%)$ & 0.38 \\
\hline Systolic blood pressure (mm Hg) & $123.3(16.1)$ & $124.6(17.7)$ & $127.1(17.5)$ & $<0.001$ \\
\hline Diastolic blood pressure $(\mathrm{mm} \mathrm{Hg})$ & $76.0(9.9)$ & $77.0(9.5)$ & $77.5(9.2)$ & 0.01 \\
\hline Low-density lipoproteins $(\mathrm{mmol} / \mathrm{L})$ & $3.1(0.9)$ & $3.2(0.9)$ & $3.4(0.9)$ & $<0.001$ \\
\hline$(\mathrm{mg} / \mathrm{dL})$ & $119.9(34.8)$ & $123.7(34.8)$ & $131.5(34.8)$ & \\
\hline High-density lipoproteins (mmol/L) & $1.3(0.3)$ & $1.3(0.3)$ & $1.3(0.4)$ & 0.42 \\
\hline$(\mathrm{mg} / \mathrm{dL})$ & $50.3(11.6)$ & $50.3(11.6)$ & $50.3(15.5)$ & \\
\hline Use of lipid-lowering medications & $21(3.3)$ & $18(2.8)$ & $16(2.5)$ & 0.20 \\
\hline Framingham risk score & $-0.8(9.9)$ & $1.5(8.9)$ & $3.7(8.0)$ & $<0.001$ \\
\hline Framingham risk category & & & & $<0.001$ \\
\hline $0-10 \%$ & $491(76.8 \%)$ & $466(72.9 \%)$ & $394(61.7 \%)$ & \\
\hline $10-20 \%$ & $75(11.7 \%)$ & $111(17.4 \%)$ & $139(21.8 \%)$ & \\
\hline$>20 \%$ & $73(11.4 \%)$ & $62(9.7 \%)$ & $106(16.6 \%)$ & \\
\hline hs-CRP level, median (IQR) (mg/L) & $1.4(0.5-3.4)$ & $1.6(0.5-3.6)$ & $1.9(0.7-4.4)$ & 0.005 \\
\hline IL-6 level, median (IQR) (pg/mL) & $1.1(0.7-1.9)$ & $1.1(0.7-1.9)$ & $1.2(0.8-2.2)$ & 0.38 \\
\hline sICAM-1 level (ng/mL) & $635.5(341.7)$ & $543.2(328.2)$ & $528.4(313.9)$ & $<0.001$ \\
\hline
\end{tabular}

Values are mean (SD) or $\mathrm{n}(\%)$ except where noted otherwise. P-values for increasing tertiles of telomere length was performed using Goodman and Kruskal's gamma statistic for categorical variables, and chi-square statistic derived from linear regression for continuous variables. Trend testing for hs-CRP and IL-6 levels was performed after log-transform due to for skew.

Abbreviations. CRP, C-reactive protein; IL-6, interleukin-6; sICAM-1, soluble intracellular adhesion molecule-1 


\section{Table 2}

Incident rates and hazard ratios for fatal and non-fatal coronary heart disease events by tertiles of telomere length (in kilo-bases)

\begin{tabular}{|c|c|c|c|}
\hline \multirow[b]{3}{*}{ Telomere length (kilo-bases) } & \multicolumn{3}{|c|}{ Tertiles of telomere length } \\
\hline & $\begin{array}{l}\text { Longest (Reference) } \\
\quad(n=639)\end{array}$ & $\begin{array}{c}\text { Middle } \\
(n=639)\end{array}$ & $\begin{array}{l}\text { Shortest } \\
(n=639)\end{array}$ \\
\hline & 5.5 to 8.2 & 5.0 to $<5.5$ & 4.1 to $<5.0$ \\
\hline Number of events & 35 & 62 & 67 \\
\hline Incidence per 1,000 person-years & 6.2 & 11.2 & 12.2 \\
\hline \multicolumn{4}{|l|}{ Hazard ratios $(95 \% \mathrm{CI})$} \\
\hline Unadjusted & 1.00 (Reference) & $1.81(1.20-2.75)$ & $1.97(1.31-2.96)$ \\
\hline Model $1 *$ & 1.00 (Reference) & $1.63(1.08-2.47)$ & $1.22(0.81-1.83)$ \\
\hline Model $2{ }^{\dagger}$ & 1.00 (Reference) & $1.64(1.07-2.50)$ & $1.30(0.86-1.97)$ \\
\hline Model $3{ }^{*}$ & 1.00 (Reference) & $1.63(1.07-2.51)$ & $1.25(0.82-1.90)$ \\
\hline
\end{tabular}

Model 1 includes adjustment for age and sex.

${ }^{\dagger}$ Model 2 includes adjustment for covariates in Model 1 plus body mass index, Framingham risk score, use of lipid-lowering medications, and physical activity.

${ }^{*}$ Model 3 includes adjustment for covariates in Model 2 plus ln CRP, ln IL6, and sICAM-1. 
Table 3

Hazard ratio for clinical events by tertiles of telomere length (in kilo-bases) and event type*

\begin{tabular}{|c|c|c|c|}
\hline \multirow[b]{3}{*}{ Telomere length (kilo-bases) } & \multicolumn{3}{|c|}{ Tertiles of telomere length } \\
\hline & $\begin{array}{c}\text { Longest (Reference) } \\
\quad(n=643)\end{array}$ & $\begin{array}{c}\text { Middle } \\
(\mathrm{n}=644)\end{array}$ & $\begin{array}{l}\text { Shortest } \\
(n=644)\end{array}$ \\
\hline & 5.5 to 8.2 & 5.0 to $<5.5$ & 4.1 to $<5.0$ \\
\hline \multicolumn{4}{|l|}{ CHD event or all-cause mortality } \\
\hline Number of events & 70 & 96 & 122 \\
\hline Hazard ratio $(95 \% \mathrm{CI})$ & 1.00 (Reference) & $1.30(0.94-1.80)$ & $1.09(0.81-1.48)$ \\
\hline \multicolumn{4}{|l|}{ All-cause mortality only } \\
\hline Number of events & 46 & 52 & 83 \\
\hline Hazard ratio $(95 \% \mathrm{CI})$ & 1.00 (Reference) & $1.11(0.73-1.69)$ & $1.12(0.77-1.62)$ \\
\hline \multicolumn{4}{|l|}{ Non-fatal CHD events only } \\
\hline Number of events & 34 & 58 & 61 \\
\hline Hazard ratio $(95 \% \mathrm{CI})$ & 1.00 (Reference) & $1.56(1.00-2.41)$ & $1.17(0.76-1.80)$ \\
\hline \multicolumn{4}{|l|}{ Fatal CHD events only } \\
\hline Number of events & 4 & 8 & 11 \\
\hline Hazard ratio $(95 \% \mathrm{CI})$ & 1.00 (Reference) & $2.05(0.61-6.92)$ & $1.79(0.56-5.72)$ \\
\hline
\end{tabular}

* Hazard ratios are adjusted for age, sex, body mass index, Framingham risk score, use of lipid-lowering medications, physical activity, ln CRP, ln IL6, and SICAM-1. 